Cornejo-Elgueta, J. \& Sánchez-Fuentes, S. (2022). El Aprendizaje y Servicio en la retención de estudiantes de ingeniería . Revista Electrónica Interuniversitaria de Formación del Profesorado, 25(1), 131-143.

\title{
El Aprendizaje y Servicio en la retención de estudiantes de ingeniería
}

\author{
Jorge Cornejo-Elgueta', Sergio Sánchez-Fuentes² \\ (1) Universidad Tecnológica Metropolitana (Chile), (2) Universidad Autónoma de Madrid \\ (España)
}

\section{Resumen}

El Aprendizaje-Servicio (en adelante ApS) se considera una metodología de enseñanza que privilegia la participación del alumnado situándolo en el rol protagónico o principal para construir su propio aprendizaje. El objetivo de este trabajo es conocer y analizar la percepción de estudiantes y docentes respecto de cómo incide la aplicación de ApS en la permanencia de estudiantes del primer ciclo formativo en carreras de computación e informática. Se ha utilizado un método mixto de investigación, con predominancia del enfoque cualitativo, recurriendo a una muestra por conveniencia de 10 docentes y 49 estudiantes de dos facultades de ingeniería de Chile. Además, también se contó con la participación de un total de 6 expertos en ApS. Los resultados obtenidos, a partir de cuestionarios autoadministrados y encuestas, y procesados mediante el software Atlas.Ti, mostraron que el ApS incide efectivamente, y de manera positiva, en el rendimiento del estudiantado. Finalmente, los autores concluyen que es necesario implementar propuestas de aprendizajes innovadoras, participativas, que combinen teoría y práctica, que logren un aprendizaje significativo en el estudiante, que lo vinculen a problemas del entorno y desarrollen un mayor sentido social.

\section{Palabras clave}

Aprendizaje-Servicio; Computación e Informática; Universidad; Deserción estudiantil. 


\title{
Learning and Service in the retention of engineering students
}

\begin{abstract}
Service-Learning is considered a teaching methodology that favors the participation of students, placing them in the leading or main role to build their own learning. The aim of this paper is to know and to analyze the perception of students and professors regarding how the application of Service-Learning affects the permanence of students of the first training cycle in computing and informatics degrees. A mixed research method has been used, with a predominance of the qualitative approach, using a convenience sample of 10 professors and 49 students from two engineering colleges in Chile. In addition, a total of 6 experts on Servie-learning also participated. The results obtained, from self-administered questionnaires and surveys, and processed were using the Atlas.Ti software, showed that SL has an effective and positive impact on student performance. Finally, the authors conclude that it is necessary to implement innovative, participatory learning proposals that combine theory and practice, that achieve significant learning in the student, that link them to environmental problems and develop a greater social sense.
\end{abstract}

\section{Key words}

Service-Learning; Computer Science; University; Students dropping.

\section{Introducción}

En el año 2006 la UNESCO señalaba que la deserción estudiantil debía ser materia prioritaria para los países miembros de la organización (IESALC-UNESCO, 2006). Según antecedentes del Ministerio de Educación de Chile alrededor del $25 \%$ de los estudiantes universitarios no continúan sus estudios después del primer año (SIES, 2019), esto afecta directamente a las instituciones de educación superior (IES) y, particularmente, al desarrollo personal y social de quienes se ven en la obligación a interrumpir un proyecto de vida. En las carreras de ingeniería esta cifra supera el 30\% (SIES, 2019). A esto se añade que, según la Organización para la Cooperación y el Desarrollo Económico, la tasa de graduación promedio de estudiantes universitarios no supera el 50\% (OCDE, 2017), lo que contribuye significativamente a la desigualdad social.

Al respecto Munizaga, et al., (2018) señalan que: "Para lograr un sistema equitativo y de inclusión social, no es suficiente con otorgar la oportunidad de ingreso a estudiantes de sectores infrarrepresentados, sino que también es necesario garantizar la permanencia, el desempeño y las posibilidades de que estos estudiantes obtengan buenos resultados." (p.5)

Entre las causas más determinantes de la deserción estudiantil en primer año de universidad se destacan: problemas vocacionales, situación económica familiar y rendimiento académico (Centro de Microdatos, 2008). Este informe señala que algunos aspectos del bajo rendimiento académico son: i) Baja motivación y problemas vocacionales; ii) debilidades académicas previas; iii) debilidades en metodologías de enseñanza y aprendizaje; iv) insatisfacción con la carrera.

En contraposición a la deserción, Donoso (2010) describe la retención estudiantil como las acciones destinadas a que el estudiante alcance los aprendizajes requeridos para permanecer en la institución y continúe estudiando. Otros autores (López, 2013; Fernández, 2009) 
mencionan respecto de la retención, que supone el porcentaje de estudiantes que estando matriculados en el primer año, continuaron sus estudios junto a sus congéneres. Fernández (2009), va un paso más allá y propone: "Los términos deserción, abandono, persistencia y permanencia se centran en el estudiante. Los dos primeros tienen connotación negativa, en tanto que los últimos son positivos. Asimismo, el término retención es positivo, pero focaliza la responsabilidad del proceso en la institución" (p.4).

La literatura indica que existen metodologías activas de apoyo a la docencia que pueden contribuir a mejorar los procesos de enseñanza aprendizaje en la educación superior, como por ejemplo: Aprendizaje Basado en Investigación (ABI), Aprendizaje Basado en Problemas (ABP), Aprendizaje Basado en Proyectos, Aprendizaje Basado en Casos, Instrucción entre Pares, Juegos de Rol, Aula Invertida, Método STEM, Aprendizaje y Servicio, etc. (Jiménez, et al., 2020).

Estas metodologías atraen el interés de instituciones y docentes, con respecto a las metodologías tradicionales, predominantes aún en la mayoría de los sistemas educativos, en donde priman criterios academicistas (Jiménez, et al., 2021). Distingue a las metodología activas, en particular a ApS, situar al estudiante en un rol protagónico de su proceso de aprendizaje proporcionándole innumerables contextos en que desarrollar los aprendizajes.

El ApS es una potente herramienta en la formación de profesionales de diversas disciplinas, como por ejemplo, en las áreas de la salud, ciencias sociales, educación e ingeniería. Sin embargo, en carreras relacionadas con computación e informática, existen muy pocas experiencias registradas. A modo de ejemplo en el libro "Aprendizaje Servicio en la educación superior chilena (Pizarro y Hasbún, 2019) que reúne las experiencias de diecisiete universidades, se constata que solamente en seis de ellas existen casos relacionados con carreras de ingeniería, y ninguna pertenece al ámbito de la computación e informática.

Finalmente, cabe señalar que este trabajo surge a partir de un estudio realizado en torno al tema Aprendizaje y Servicio, y retención estudiantil en ingeniería, llevado a cabo en dos facultades de dos universidades chilenas. A partir del amplio material recopilado a través de entrevistas a estudiantes, docentes y expertos en ApS en educación superior en Chile, se observó que el rendimiento académico se encontraba estrechamente relacionado con la forma en que el profesorado entregaba los contenidos. Al respecto, un estudio realizado por Castillo-Montes y Ramírez-Santana (2020) plantea que "el uso de metodologías activas contribuye a fomentar el aprendizaje activo, mejorando la satisfacción y calificaciones de los estudiantes, especialmente de aquellos con menor desempeño académico".

Por todo ello, el objetivo de este estudio es valorar el desafío que representa para la metodología ApS contribuir a mejorar en la retención de estudiantes de carreras de ingeniería de Computación e Informática.

\section{Metodología}

\section{Procedimiento}

La metodología utilizada fue el método mixto de investigación con predominio del enfoque cualitativo, para esto se escogió una muestra por conveniencia, en base a que se necesitaba personas que hubiesen tenido contacto directo con la metodología ApS. El modelo de investigación que se utilizó fue de carácter secuencial, prevaleciendo la mirada de índole cualitativa, descriptiva, interpretativa y con diseño de tipo micro etnográfico. Se buscó apreciar la percepción que existe en estudiantes de ingeniería y docentes, así como de expertos. Las percepciones reflejan la interacción de los elementos individuales y las 
características y procesos de la organización, también indica que son muy variadas y en parte se determinan social y culturalmente (Hernández, et al., 2014).

\section{Muestra}

Estuvo conformada por 10 docentes y 49 estudiantes de dos facultades de ingeniería, de una universidad pública y otra privada de Santiago de Chile. Respecto de los estudiantes, 13 fueron mujeres, mientras que los 36 restantes fueron hombres, y la edad de todos los participantes estuvo comprendida entre los 21 y los 25 años. En cuanto a los profesores, 7 de ellos fueron hombres y 3 fueron mujeres. Todos los docentes superan los 40 años de edad, siendo la franja entre 40 y 50 la más habitual.

En cuanto a las universidades, las instituciones fueron la Universidad Tecnológica Metropolitana y la Universidad Central de Chile. Para el objetivo de la investigación se recurrió a las respectivas Facultades de Ingeniería de ambos centros de educación superior, que se encuentran en Santiago de Chile.

Además, también se contó con la participación de un total de 6 expertos en ApS. De entre ellos, 4 fueron mujeres y 2 eran hombres. La media de edad fue superior a los 40 años y todos tenían experiencia en docencia, gestión y desarrollo de programas de ApS.

La unidad de análisis, la integraron:

- Docentes de ingeniería, con al menos diez años de docencia universitaria, que han participado en asignaturas con proyectos de ApS.

- Estudiantes de ingeniería, con al menos dos años de permanencia en carreras de un plan regular de pregrado tradicional diurno, que han tenido asignaturas con proyectos de ApS.

- Expertos de reconocido prestigio en ApS, académicos y académicas, con más de diez años aplicando ApS en diferentes IES.

\section{Instrumentos}

La recolección de datos se llevó a cabo mediante entrevistas semiestructuradas. Para el estudio cualitativo, las preguntas fueron aplicadas a 11 estudiantes y a todos los docentes, donde las diez preguntas estuvieron distribuidas en dos categorías: deserción estudiantil y metodologías de aprendizaje. Por otro lado, para los expertos el cuestionario contó con seis preguntas relacionadas directamente con el aporte de la metodología ApS en carreras de ingeniería. Para el estudio cuantitativo, la encuesta a se aplicó a 38 estudiantes y fue desarrollada a través de doce preguntas en un rango de entre "Muy en desacuerdo" hasta "Muy de acuerdo", en relación a la incidencia de la metodología ApS en la retención de estudiantes de ingeniería.

\section{Criterios éticos}

Se recurrió al consentimiento informado, tomando como base la propuesta de Taylor y Bogdan (1987), que dice relación con proteger el anonimato de los participantes y en segundo lugar, el investigador debe evitar interferir en las actividades de los investigados. Con esto se garantizó al participante el anonimato y la confidencialidad de los datos proporcionados. Además, se solicitó los correspondientes permisos a las autoridades de cada grupo de entrevistados, dando a conocer los objetivos de la investigación, los derechos y responsabilidades de los participantes como del investigador responsable. 


\section{Análisis de datos}

La primera fase consistió en la elaboración de las preguntas, que fueron validadas por jueces expertos, y posteriormente sometidas a una prueba piloto. La segunda fase se centró en la aplicación de las entrevistas en los diferentes escenarios.

Para la etapa cualitativa se definieron dos escenarios que se abordaron en paralelo, para estudiantes y docentes, de cada una de las universidades. Por lo tanto se utilizaron dos cuestionarios diferentes dependiendo de cada estamento, para luego con los datos recabados generar las matrices de codificación abierta, axial y selectiva.

Los resultados proporcionados por docentes y estudiantes, se contrastaron con las respuestas de expertos en ApS, recogidas también mediante entrevistas semiestructuradas.

Una vez recolectadas las narraciones de estudiantes, docentes y expertos en ApS, se recurrió el software Atlas.Ti para el análisis de los datos, principalmente debido a su fácil manejo y que facilita la localización y recuperación de datos de manera sencilla. A partir del análisis de discurso se determinaron diferentes subcategorías emergentes que permitieron agrupar las percepciones de los sujetos de estudio.

Para la parte cuantitativa se recurrió a una encuesta, solo a estudiantes, con el fin de corroborar la percepción de sus condiscípulos que participaron en la fase cualitativa.

\section{Resultados}

Se presentan en dos apartados: primero se describen los resultados obtenidos para el estudio cualitativo, y en el segundo, en torno al estudio cuantitativo.

\section{Estudio cualitativo}

Al comparar los resultados obtenidos de las entrevistas a estudiantes y docentes de ambas instituciones con las respuestas de los expertos en ApS, se observaron comentarios similares en cada una de las categorías tratadas:

- Estudiantes, docentes y expertos destacan respecto a la metodología ApS, que:

- Contribuye a la retención del estudiantado por medio de: la motivación, el rol protagónico del estudiante, aprender haciendo y experimentando, tomando decisiones, etc.

- Es un aporte para la formación profesional del ingeniero, dado que es importante el acercamiento a la realidad.

- El cambio de paradigma es un elemento interesante que surge en el proceso de enseñanza aprendizaje, como por ejemplo: el rol del docente, las evaluaciones, el desarrollo de las capacidades individuales de los estudiantes, etc.

- $\quad$ Expertos y docentes señalan, que:

- Es necesario contar con el apoyo institucional a esta propuesta metodológica, no tan solo con recursos, sino también con reconocimiento a quienes participan.

- La capacitación docente en la aplicación de esta metodología es primordial para conseguir el éxito del proyecto, y minimizar la probabilidad de riesgos negativos. 
- Las competencias y habilidades "blandas" que provee la aplicación de la metodología ApS, tributan al Perfil de egreso del estudiante de ingeniería.

- Aprueban la incorporación de esta metodología en el primer ciclo formativo como elemento motivacional. Sin embargo, hacen notar que es necesario realizar un diagnóstico previo de las competencias y conocimientos del estudiante, para no perder de vista los objetivos de aprendizaje, y no cometer errores desvirtuando el fin de la metodología.

- El concepto de ciudadanía surge fuertemente por parte de las y los expertos, no tanto así por parte de docentes y estudiantes, esto probablemente se debe a la amplitud de visión de quienes cuentan con más años trabajando con esta metodología.

Los resultados obtenidos en esta fase se sintetizan en la Tabla 1, en donde se incluyen los temas relevantes que surgieron a partir de entrevistas a los diferentes actores.

\section{Tabla 1.}

Resultados cualitativos

Nota: 1: Estudiante; 2: Docente; 3: Experto

\begin{tabular}{|c|c|c|c|}
\hline Tópico & 1 & 2 & 3 \\
\hline $\begin{array}{l}\text { ApS contribuye a la retención del estudiantado por medio de la } \\
\text { motivación, el rol protagónico del estudiantado, aprender haciendo } \\
\text { y experimentando, tomando decisiones, etc. }\end{array}$ & $x$ & $x$ & $x$ \\
\hline $\begin{array}{l}\text { La capacitación a docentes es primordial para conseguir el éxito del } \\
\text { proyecto que se emprenda por medio de esta metodología. }\end{array}$ & & $x$ & $x$ \\
\hline $\begin{array}{l}\text { Es necesario contar con el constante apoyo institucional a esta } \\
\text { propuesta metodológica. }\end{array}$ & & $x$ & $x$ \\
\hline $\begin{array}{l}\text { ApS es un aporte para la formación de ingenieros e ingenieras, a } \\
\text { través del acercamiento a la realidad, el trato con el socio } \\
\text { comunitario, y el enfrentar problemas reales y no hipotéticos. }\end{array}$ & $x$ & $x$ & $x$ \\
\hline $\begin{array}{l}\text { El acercamiento a la realidad es importante en la formación } \\
\text { profesional de ingenieros e ingenieras. }\end{array}$ & $x$ & $x$ & $x$ \\
\hline $\begin{array}{l}\text { Competencias y habilidades "blandas" que provee la aplicación de } \\
\text { ApS, tributan al Perfil de egreso del estudiante de ingeniería }\end{array}$ & & $x$ & $x$ \\
\hline $\begin{array}{l}\text { Cambio de paradigmas en el proceso de enseñanza aprendizaje, en } \\
\text { torno al rol del docente, del estudiante, evaluaciones y otras. }\end{array}$ & $x$ & $x$ & $x$ \\
\hline $\begin{array}{l}\text { Conocimiento de ApS que debe poseer el docente, con el fin de } \\
\text { alcanzar el éxito en el proceso de aprendizaje. }\end{array}$ & $x$ & $x$ & $x$ \\
\hline $\begin{array}{l}\text { El concepto de ciudadanía surge fuertemente por la vinculación con } \\
\text { el medio y el compromiso con la comunidad. }\end{array}$ & & & $x$ \\
\hline $\begin{array}{l}\text { Aprueba la incorporación de ApS en el primer ciclo formativo como } \\
\text { elemento motivacional para evitar la deserción. }\end{array}$ & $x$ & $x$ & $x$ \\
\hline
\end{tabular}


Aquí es posible observar como algunos temas estuvieron presentes en las respuestas de los tres estamentos consultados, más allá de si pertenecen a una u otra facultad o si se relacionan o no con las instituciones en estudio, como es el caso, en que los tres aprueban la incorporación de la metodología ApS en el primer ciclo formativo como elemento motivacional para evitar la deserción.

\section{Estudio cuantitativo}

Los resultados que se presentan surgen a partir de las encuestas realizadas a 38 estudiantes de ambas facultades:

- La percepción respecto a la deserción estudiantil en carreras de ingeniería se encuentra relacionada con las metodologías de enseñanza utilizadas por docentes.

- La retención del alumno está en relación directa con la forma como aprende en clases.

- Se asigna gran importancia a aquellas clases en que la participación es activa o participativa.

- La mayoría considera que la metodología ApS, da la oportunidad de aprender de manera diferente.

- La metodología ApS sería un buen aporte para la retención de estudiantes de primeros años de la carrera.

- Las asignaturas en donde se aplica la metodología ApS estimulan el deseo de llegar más lejos en sus intereses académicos.

- Existe satisfacción por haber cursado asignaturas con ApS, motivo por el que las recomendarían a otros estudiantes.

- En cuanto a que "esta metodología supondría una carga de trabajo adicional”, a la mayoría de las y los encuestados esto le es indiferente.

- Respecto a que "las asignaturas con ApS ayudan a consolidar los conocimientos teóricos", la mayoría del estudiantado consultado se encuentra de acuerdo con esta afirmación.

- En relación a si "el interés por la carrera ha aumentado como resultado de haber cursado una asignatura con ApS", las y los estudiantes, le restan importancia a esto debido principalmente a que las asignaturas con ApS se han impartido en cursos de tercer y cuarto año de carrera, donde se asume que el estudiante tiene muy claro el Perfil de egreso.

Los resultados de esta encuesta se encuentran en la Tabla 2.

Tabla 2.

Resultados cuantitativos encuesta estudiantes de ambas instituciones

Escala: 1: Muy en desacuerdo; 2: En desacuerdo; 3: Indiferente; 4: De acuerdo; 5: Muy de acuerdo

\begin{tabular}{llllllllllll}
\hline Variables & & $\mathbf{1}$ & $\mathbf{2}$ & & $\mathbf{3}$ & & $\mathbf{4}$ & & $\mathbf{5}$ \\
\cline { 2 - 9 } & $\mathrm{N}$ & $\%$ & $\mathrm{~N}$ & $\%$ & $\mathrm{~N}$ & $\%$ & $\mathrm{~N}$ & $\%$ & $\mathrm{~N}$ & $\%$ \\
\hline $\begin{array}{l}\text { 1. Deserción estudiantil se relaciona } \\
\text { con las metodologías de enseñanza. }\end{array}$ & 4 & $11 \%$ & 3 & $8 \%$ & 18 & $47 \%$ & 13 & $34 \%$ & 0 & $0 \%$
\end{tabular}


2. La retención está en relación

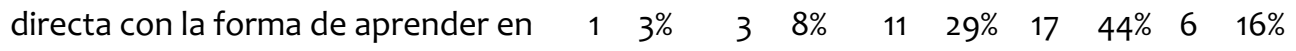
clases.

3. La retención se relaciona con la $\begin{array}{llllllllllll}\text { forma en que el profesor hace su } & 1 & 3 \% & 4 & 11 \% & 8 & 20 \% & 19 & 50 \% & 6 & 16 \%\end{array}$ clase.

4. Una clase amena y productiva es aquella en la que participo

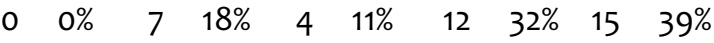
activamente.

5. ApS da la oportunidad de aprender de manera diferente.

$\begin{array}{llllllllll}1 & 3 \% & 0 & 0 \% & 6 & 16 \% & 15 & 39 \% & 16 & 42 \%\end{array}$

6. ApS sería un aporte para la

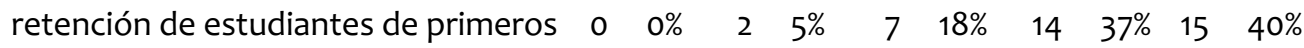
años.

7. ApS estimula el deseo de llegar más lejos en mis intereses académicos.

8. Recomendaría asignaturas donde se aplica ApS.

9. Me encuentro satisfecho de haber cursado asignaturas con ApS.

10. La carga de trabajo de asignaturas con ApS es adecuada.

11. Las asignaturas con ApS ayudan a consolidar los conocimientos teóricos.

12. Mi interés por la carrera aumentó al cursar asignaturas con ApS.

De manera gráfica (véase Figura 1) es posible observar que el predominio en las respuestas se encuentra en las opciones "De acuerdo" y "Muy de acuerdo" en aquellas preguntas que se relacionan con la metodología ApS, mientras que el predominio en torno a las variables retención y metodología se concentra en las opciones "Indiferente" y "De acuerdo".

\section{Discusión}

Se observa que la percepción de las y los entrevistados es muy optimista en cuanto al aporte de la metodología ApS a la retención del estudiante de primer y segundo año, lo que no deja de ser significativo y esperanzador; sin embargo, la implementación de esta metodología requiere de una preparación previa exhaustiva y rigurosa por parte del docente, debido a que debe tratar con estudiantes que aún no se desconectan de un sistema educativo rígido y autoritario que le indica que debe hacer paso a paso, por lo tanto ese estudiante aún no se encuentra preparado para asumir desafíos en donde debe tomar decisiones y responder de manera responsable ante el socio comunitario. Por lo que es necesario contar con docentes que posean formación pedagógica y/o cuenten con habilidades que le permitan "encantar" al estudiante con su carrera profesional. Preparar docentes que cumplan esta función es un 
desafío para las IES. Contar con una unidad o centro de apoyo a la docencia, podría facilitar el éxito en la implementación de esta metodología. Tal como lo mencionó uno de los expertos: "Es necesario disponer de unidades de apoyo a nivel institucional que faciliten la capacitación docente, selección de socios comunitarios y seguimiento para la mejora continua en aspectos curriculares, pedagógicos y vinculación con el medio".

Figura 1

Resultados cuantitativos encuesta estudiantes de ambas instituciones

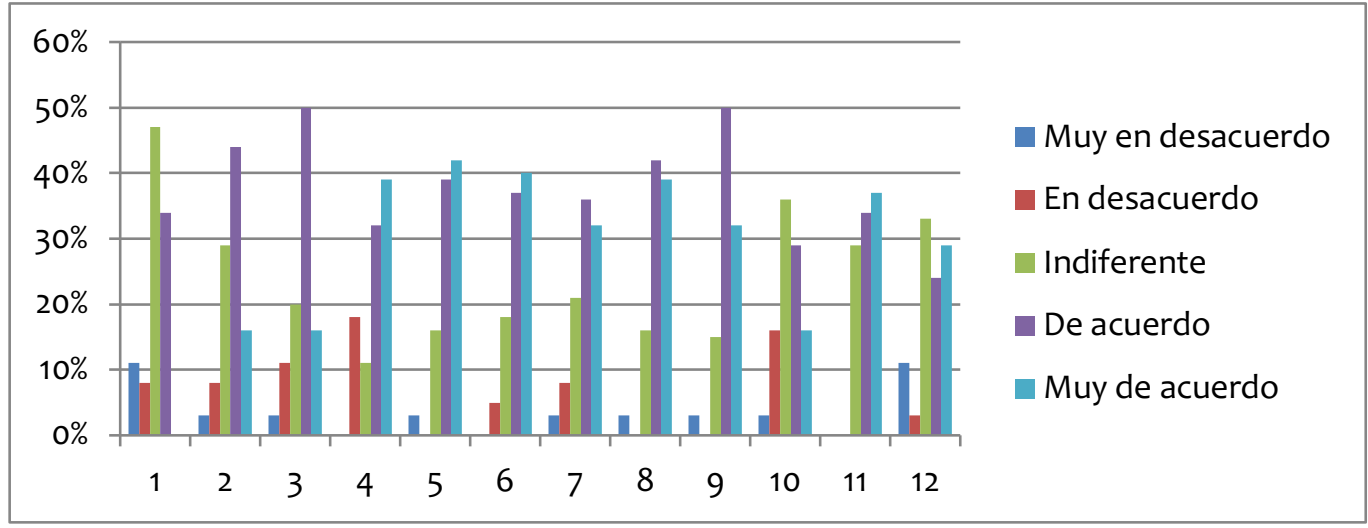

El planteamiento que surge a partir de los antecedentes proporcionados por las y los estudiantes, referido a la posibilidad de auto-superación es un hallazgo interesante. Si bien los estudiantes plantean propuestas al respecto, como por ejemplo "superarse a sí mismo para satisfacer las necesidades del cliente", hay dos aspectos que sobresalen y que es importante considerar respecto a la adquisición del conocimiento: la aplicación de metodologías activas correctamente utilizadas por parte del docente, pueden hacer la diferencia en el proceso de aprendizaje del estudiante a través de actividades concretas; y en segundo lugar, destacar la actitud de ellos mismos hacia el logro de sus objetivos a través de la motivación. Capponi (2019) plantea que no hay reemplazo para la experiencia, es importante la experiencia directa con el mundo real, hacer las cosas por sobre teorizar, por lo que debemos construir nuestros propios recursos mentales a partir de lo concreto para enfrentarnos a la sociedad con éxito.

El carácter práctico de esta metodología puede ser un incentivo para los estudiantes, sin embargo, no se debe descuidar los aspectos teóricos, primero debe existir un contexto teórico sólido que permita al estudiante poner en práctica lo aprendido de manera correcta, dando cabida al error, pero valorando el aprendizaje adquirido, para esto la metodología provee de un proceso de Reflexión participativa, que facilita darse cuenta de manera crítica de los avances del proyecto o sus traspiés.

La metodología ApS sirve al docente para situarse en un cambio de paradigma respecto de la docencia, cediendo el protagonismo al estudiante, a quienes debe movilizar. Este cambio de paradigma de una enseñanza tradicional a una metodología activa, más participativa y dinámica, obliga al docente a manejar herramientas modernas de índole pedagógica y psicológica. Por lo tanto, si se quiere co-construir en la generación de saberes dinámicos, reflexivos, críticos y de producción de conocimiento, se debe estar dispuesto no solo a aceptar este cambio de paradigma sino también a prepararse ante este desafío. 
En el transcurso de la investigación se pudo apreciar que la metodología ApS se presenta como una oportunidad para mejorar los indicadores de retención, la formación docente y la innovación en la educación superior. Sin embargo, esto requiere coordinar múltiples esfuerzos, y llevar a cabo un programa de largo alcance, con el apoyo sistemático de la institución, como todo proceso de mejora continua, pausado y planificado, incorporado en el currículo, que permita un avance en lo académico con sólidas bases, y de paso establezca estrechas relaciones con la comunidad.

La aplicación de la metodología si bien se centra en el estudiante y su aprendizaje, también debe considerar a la comunidad a la que brinda el servicio. Así como el ApS es una innovación que puede conducir a una experiencia enriquecedora para las y los estudiantes, también debe serlo para la comunidad, de manera que para ambas partes sea una experiencia satisfactoria, no tan sólo desde el punto de vista material, sino también emocional.

Uno de los aspectos que trajo a colación la investigación dice relación con los obstáculos que se presentan en la aplicación de la metodología, por parte de docentes y expertos consultados, entre estos surge fuertemente el tema de la carga de trabajo adicional que supone la metodología, hecho que es una realidad. Un proyecto de ApS requiere contar con recursos mínimos necesarios para ponerlo en práctica, en este caso no se trata de presupuesto, sino de tiempo asignado a los docentes para planificar y preparar las clases, las reuniones previas con los socios comunitarios, los cierres de actividad y los informes finales. Muchas de las veces estos aspectos no se consideran y son aportados por el docente, lo que en cierta medida desmotiva cambiar de paradigma y se mantiene con la clase tradicional. Se hace mención respecto a algunos incentivos al docente por parte de la institución, como por ejemplo: capacitaciones, participación en congresos, etc. lo que en cierta medida compensa el esfuerzo y destaca su labor, pero aún falta mucho por hacer al respecto, como por ejemplo certificar a los docentes en ApS, valorar la metodología como una herramienta para la formación de mejores profesionales, y como un aporte a la retención estudiantil. Por mientras, si un docente desea trascender en la formación de sus estudiantes debe llevar a cabo este esfuerzo asumiendo costos personales y profesionales.

\section{Conclusiones}

En palabras de los propios entrevistados, la metodología ApS es importante en la formación en ingeniería, debido a que moldea el carácter y aptitudes necesarias para que el estudiante sea capaz de abordar un problema, reconocerlo, estudiarlo y darle solución. De esta forma el estudiantado se interesa de mayor manera en las asignaturas de la carrera y se forma un ingeniero integral. A esto se puede agregar, que el uso de la metodología ApS, es una oportunidad de aprender de experiencias in situ y desarrollar habilidades blandas que serán necesarias para la vida laboral futura. ApS es una herramienta que permite visualizar mejoras, los enfrenta a la realidad y afianza sus conocimientos con un compromiso social. También, desarrolla las competencias declaradas en el Perfil de egreso de la carrera.

Duffy et al., (2008) señala que el ApS es eficaz en un gran número de medidas cognitivas y afectivas en estudiantes de diferentes ingenierías, logra un mejor conocimiento de la materia del curso, el aprendizaje cooperativo y el reclutamiento de grupos sub-representados en ingeniería; también, que esta metodología conduce a una mejor retención de estudiantes, además de ayudar a cumplir con los conocidos criterios ABET [Nota 1]. Agrega que los proyectos que utilizan ApS tienen el potencial de asegurar que las y los estudiantes aprendan, además de garantizar que tengan la capacidad de aplicar la ingeniería al diseño y análisis de sistemas y experimentos. Además, que la adecuación del ApS a la ingeniería se debe 
principalmente a que su aplicación puede realizarse de manera relativamente sencilla a problemas de ingeniería común y posibilita su integración en asignaturas y currículos. (p.19).

Oakes (2004) concuerda en que la metodología ApS se encuentra bien posicionada para apoyar a los programas de ingeniería en torno a los desafíos educativos actuales que a veces son difíciles de abordar en los cursos tradicionales, también señala que el aprendizaje-servicio ofrece muchas oportunidades para trabajar los programas acreditados bajo los Criterios de Ingeniería EC 2000 de ABET. (p.11).

La percepción de aprendizaje por parte de las y los estudiantes, es significativamente mayor en aquellas asignaturas donde se aplicó la metodología ApS, en donde la práctica jugó un rol importante, y el estudiante fue protagonista. Es posible afirmar a partir de esto, que producto de lo mismo el estudiante se logra motivar y entusiasmar por el trabajo ingenieril desde el comienzo de su carrera, posibilitando una mejora en los índices de retención, y como consecuencia mejorar la calidad del proceso de aprendizaje en las instituciones. Resultados que se encuentran en concordancia con los hallazgos observados por Cea y Muñoz (2017): “... desde el punto de vista del docente, con los proyectos de aprendizaje servicio no sólo se logran los aprendizajes declarados en el curso, sino que también se desarrollan otras habilidades personales e interpersonales. Entre ellas se aprecia un claro aumento del compromiso del estudiante con su aprendizaje y la comunidad. Trabajan con mayor responsabilidad y rigurosidad tanto a nivel individual como grupal. Comprenden mejor la importancia de la comunicación oral, la adaptación al cambio, la coordinación y gestión de recursos. Además, adquieren la confianza suficiente para realizar prácticas de verano, comprenden mejor el rol del Ingeniero Civil y de los profesionales del área..., como también culturas organizacionales y sociales diferentes" (p.136).

En relación a lo anterior, Oakes (2004) agrega que entre los beneficios que ApS entrega a los estudiantes de ingeniería están el desarrollo de experiencias para el trabajo en equipos multidisciplinarios, el desarrollo de habilidades comunicacionales, el vincularse con la solución de problemas reales del entorno y entender su rol y responsabilidad como ingenieros en la sociedad. (p.11)

Un factor clave y determinante es el cuerpo docente, su conocimiento de la metodología y la preparación de la actividad, si se quiere que el aprendizaje realmente ocurra. Esto requiere de una profunda comprensión de lo que se pretende con la metodología y lo que esta puede significar en el proceso de aprendizaje, y su desarrollo como persona.

En palabras de Pérez (2005) el profesor "necesita adquirir nuevos elementos y dominios en su quehacer docente por lo que precisa de formación metodológica en estrategias y técnicas innovadoras, capaz de dar respuesta a esta nueva orientación" (p.156). Esto quiere decir que los docentes en la educación superior deben ir más allá de la transmisión usual de conocimientos como planteaba Freire (1970) con su educación bancaria, sino que debe orientarse a aplicar nuevas metodologías que incentiven en el estudiantado generar nuevos conocimientos a partir de la motivación por el saber. Como lo indica Pérez (2005), se requiere que el docente sea un agente que enseñe a aprender al alumno, más que, indicarle lo "que debe hacer".

En base al estudio realizado es posible plantear que la incorporación de la metodología ApS en los primeros años de carrera en ingeniería sería muy bien recibida. Proyectando con esto, el logro de convenientes beneficios para la institución, docentes, estudiantes y sus familias, contribuyendo significativamente a la calidad de los programas de educación y a la retención estudiantil. A esto se debe sumar los múltiples beneficios que la metodología le aporta al estudiante de ingeniería, como señala Oakes (2014): Aplicaciones reales con oportunidad 
práctica; experiencia en planificación y gestión de proyectos; $y$, experiencia de diseño realista, a largo plazo y de principio a fin.

En función del contexto actual en que se encuentra nuestra sociedad, se hace necesario implementar propuestas de aprendizajes con mayor sentido social, que mejore el vínculo entre la institución y el entorno, a través de la formación de mejores ciudadanas y ciudadanos. Es aquí donde el ApS puede ser un instrumento de utilidad para que las instituciones avancen hacia la excelencia en la formación de profesionales responsables, reflexivos y deliberantes.

\section{Notas}

[1] Agencia norteamericana considerada líder mundial en el aseguramiento de la calidad para programas del área, mediante su proceso de acreditación, garantiza que el programa impartido por la universidad, prepara egresados que cumplen con los estándares globales de la profesión.

\section{Referencias}

Aramburuzabala, P.; Garcia, R.; y Elvias, S. (2013). Educación desde y para la Justicia Social: una Experiencia de Aprendizaje-Servicio en la Formación de Maestros. Variables psicológicas y educativas para la intervención en el ámbito escolar. En: M.C. PérezFuentes y M.M. Molero (Coords.), Variables psicológicas y educativas para la intervención en el ámbito escolar (pp. 257-265). Almería: Asociación Universitaria de Educación y Psicología.

Batlle, R. (2011). ¿De qué hablamos cuando hablamos de aprendizaje-servicio? Crítica, 972(61), 49-54.

Capponi, R. (2019). Felicidad sólida: Sobre la construcción de una felicidad perdurable. Caligrama.

Castillo-Montes, M., y Ramírez-Santana, M. (2020). Experiencia de enseñanza usando metodologías activas, y tecnologías de información y comunicación en estudiantes de medicina del ciclo clínico. Formación universitaria, 13(3), 65-76. http://dx.doi.org/10.4067/S0718-50062020000300065

Cea, P., González, F., y Muñoz, M. (2015). Aprendizaje servicio en Ingeniería Civil de la UCSC: Experiencia del curso de topografía. RIDAS, Revista Iberoamericana de Aprendizaje Servicio, 1, 132-137. http://doi/10.1344/RIDAS2015.1.8

Centro de Microdatos. (2008). Informe final: "Estudios sobre las causas de la deserción universitaria". Universidad de Chile, Departamento de Economía.

Donoso, S. (2010). Retención de estudiantes y éxito académico en la educación superior: análisis de buenas prácticas. Instituto de Investigación y Desarrollo Educacional, [Tesis doctoral, Universidad de Talca]. https://www.cned.cl/proyecto-deinvestigacion/retencion-de-estudiantes-y-exito-academico-en-la-educacion-superior

Duffy, J., Moeller, W., Kazmer, D., Crespo, V., Barrington, L., Barry, C., y West, C. (2008). Service-Learning Projects in Core Undergraduate Engineering Courses. International Journal for Service Learning in Engineering. 3(2), 18-41. 
Fernández, N. (2009). Retención y persistencia estudiantil en instituciones de educación superior: una revisión de la literatura. Paradigma, 30(2), 39-62.

Freire, P. (1970). Pedagogía del oprimido. Siglo XXI.

Furco, A. y Billig, S. (2002). Service learning: the essence of pedagogy. IAP.

Hernández, R., Fernández, C. y Baptista, M. P. (2014). Metodología de la investigación. McGraw-Hill.

IESALC-UNESCO. (2006). La Metamorfosis de la educación superior. Informe sobre la Educación Superior de América Latina y el Caribe 2000-2005.

Jiménez, D., Sancho, P., y Sánchez, S. (2021). Estudio acerca de las opiniones del profesorado universitario en la Región de Murcia sobre la formación de métodos activos. Revista Electrónica Interuniversitaria de Formación del Profesorado, 24(2). https://doi.org/10.6018/reifop.444381

Jiménez, D., González, J. J., y Tornel, M. (2020). Metodologías activas en la universidad y su relación con los enfoques de enseñanza. Profesorado, Revista de Currículum y Formación del Profesorado, 76-94. https://doi.org/10.30827/profesorado.v24i1.8173

López, L. (2013). La retención y la experiencia de aprendizaje De los estudiantes en el primer año de ingeniería. [Tesis doctoral, Pontificia Universidad Católica de Chile].

Munizaga, F., Cifuentes, M., y Beltrán, A. (2018). Retención y abandono estudiantil en la Educación Superior Universitaria en América Latina y el Caribe: Una revisión sistemática. Archivos Analíticos de Políticas Educativas, 26(61). http://dx.doi.org/10.14507/epaa.26.3348

Oakes, W. (2004). Aprendizaje-servicio en ingeniería: una guía de recursos. Educación superior. https://digitalcommons.unomaha.edu/slcehighered/165

Organización para la Cooperación y el Desarrollo Económicos. (2017). Evaluaciones de Políticas Nacionales de Educación: Educación en Chile. http://archivos.agenciaeducacion.cl/Educacion_en_Chile_OCDE_Nov2017.pdf

Pérez, M. (2005). Rol docente y pedagogía activa en la formación universitaria. La enseñanza centrada en el aprendizaje del alumno. Adaptación del programa al EEES. Revista Humanismo y Trabajo Social. 4, 153-175.

Pizarro, V., y Hasbún, B. (2019). Aprendizaje Servicio en la educación superior chilena. Ediciones CEA-FEN Universidad de Chile.

Puig, J. M., Batlle, R., Bosch, C., y Palos, J. (2007). Aprendizaje servicio. Educar para la ciudadanía. Ministerio de Educación y Ciencia. Octaedro.

Servicio de Información de Educación Superior. (2019). Informe de Retención de 1er año de Pregrado. https://www.mifuturo.cl/informes-retencion-de-primer-ano/

Taylor, S. J. y Bogdan, R. (1987). Introducción a los métodos cualitativos de investigación. La búsqueda de significados. Paidós. 